\title{
A Fatal Case of a Large Abdominal Wall Muscle Hematoma Secondary to Low-Molecular-Weight Heparin Injections
}

\author{
Mi Yeon Kim, M.D., Hyeon Ju Kang, M.D., Min Sun Ryu, M.D., Seo Woo Kim, M.D., Yon Ju Ryu, M.D., Ph.D., Seok Jeong Lee, M.D., \\ Jin Hwa Lee, M.D., Ph.D., Jung Hyun Chang, M.D., Ph.D., and Ji Young Hwang, M.D., Ph.D.*
}

Division of Pulmonary and Critical Care Medicine, Departments of Internal Medicine, and Radiology, Ewha Medical Center and Ewha Medical Research Institute, Ewha Womans University School of Medicine, Seoul, Korea

The use of low-molecular-weight heparin $(\mathrm{LMWH})$ can lead to major life threatening complications, including hematomas. Abdominal wall muscle hematomas are rarely fatal, and encompass a wide spectrum of severity depending on size, etiology, and associated complications; but because of their rarity may be misdiagnosed clinically. We report a fatal case of an 80 -year-old female who received LMWH after an episode of pulmonary thromboembolism and was subsequently diagnosed with a large right abdominal wall hematoma complicated with hypovolemic shock and acute kidney injury.

Key Words: hematoma; hypovolemic shock; low-molecular-weight heparin.

Low-molecular-weight heparin (LMWH) is increasingly used and has numerous indications, such as atrial fibrillation, acute coronary syndrome, thrombosis, and pulmonary embolism.[1,2] Moreover, LMWH is widely used to prevent deep-vein thrombosis in patients undergoing hip or knee surgery that require prolonged bed rest.[3] While the cost is a higher than that of comparable oral medications, LMWH is effective and safe for use in outpatient settings after proper patient education because it is unnecessary to check prothrombin time (PT) with the international normalized ratio (INR) or activated partial thromboplastin time required of coumadin and heparin. Although the rate of severe adverse reactions is relatively low compared to other oral agents, LMWH can cause lethal bleeding complica-

Received on October 4, 2013

Revised on February 3, 2014

Accepted on February 5, 2014

Correspondence to: Yon Ju Ryu, Division of Pulmonary and Critical Care Medicine, Department of Internal Medicine, Ewha Medical Center and Ewha Medical Research Institute, Mokdong Hospital, Ewha Womans University School of Medicine, 1071 Anyangcheon-ro, Seoul 158-710, Korea

Tel: +82-2-2650-2840, Fax: +82-2-2650-2559

E-mail: medyon@ewha.ac.kr tions, which may lead to death, including those from abdominal wall hematomas.[4] An abdominal wall hematoma under LMWH use is an uncommon condition, but it can occur particularly in patients with thin abdominal walls, such as elderly females or patients with a low body mass index. The risk is also greater with abdominal wall straining due to coughing or vomiting, in pregnancy, and in individuals taking other antiplatelet agents and/or anticoagulants.[5-7]

We describe a fatal case of an 80-year-old female recently diagnosed with a cerebral infarction, who was undergoing LMWH therapy for pulmonary thromboembolism and deep vein thrombosis, and who presented with an acute palpable abdominal mass in hypovolemic shock. She was diagnosed with a large right abdominal wall hematoma. This is the first such case described in Korea.

\section{CASE REPORT}

An 80-year-old female had collapsed and had been found on the floor, before being diagnosed with cerebral infarction at another hospital, 2 weeks before being seen at our hospital. She had complained of left-sided paralysis with dysarthria and the

cc This is an Open Access article distributed under the terms of the Creative Commons Attribution Non-Commercial License (http://creativecommons.org/ licenses/by-nc/3.0/) which permits unrestricted non-commercial use, distribution, and reproduction in any medium, provided the original work is properly cited.

(c) 2014 Korean Society of Critical Care Medicine 

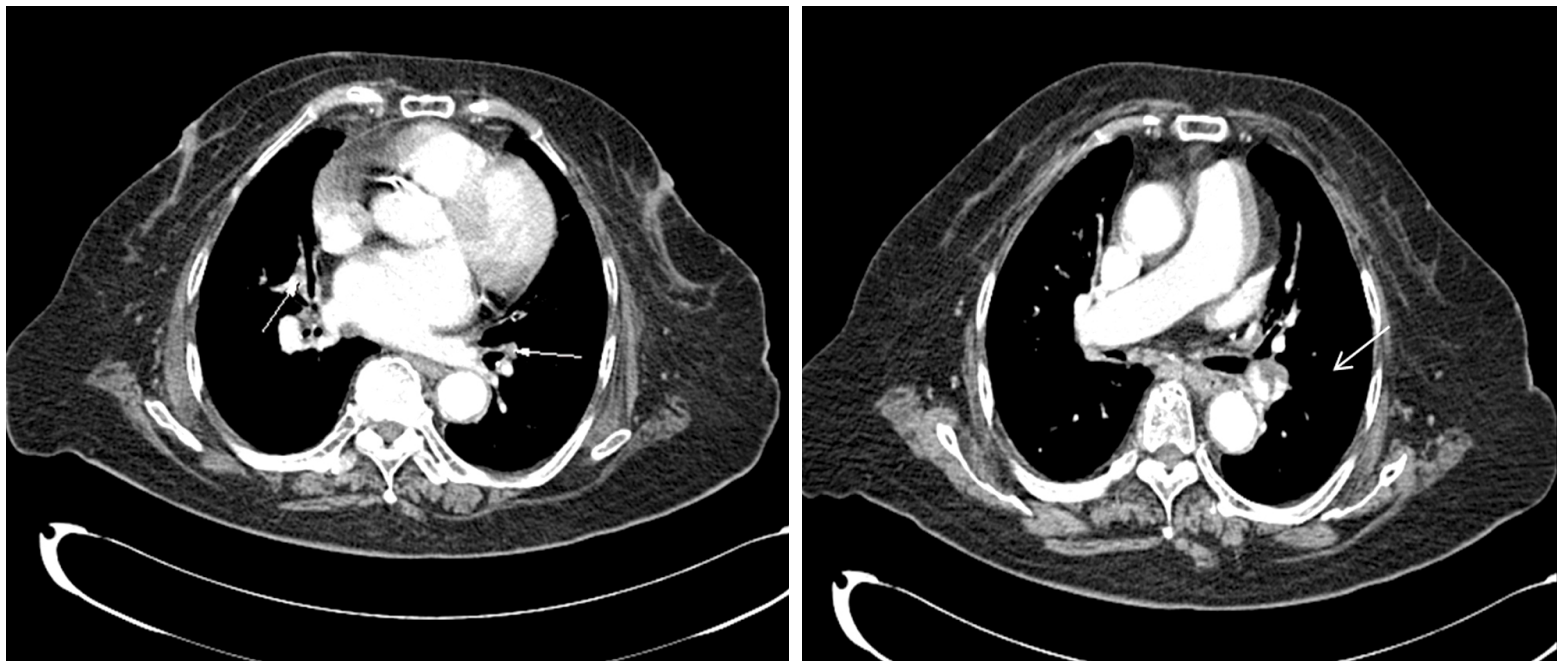

Fig. 1. Chest computed tomography (CT) scans. Chest CT scans show pulmonary thromboembolism (arrows) in left interlobar artery and multiple segmental arteries in right lung.
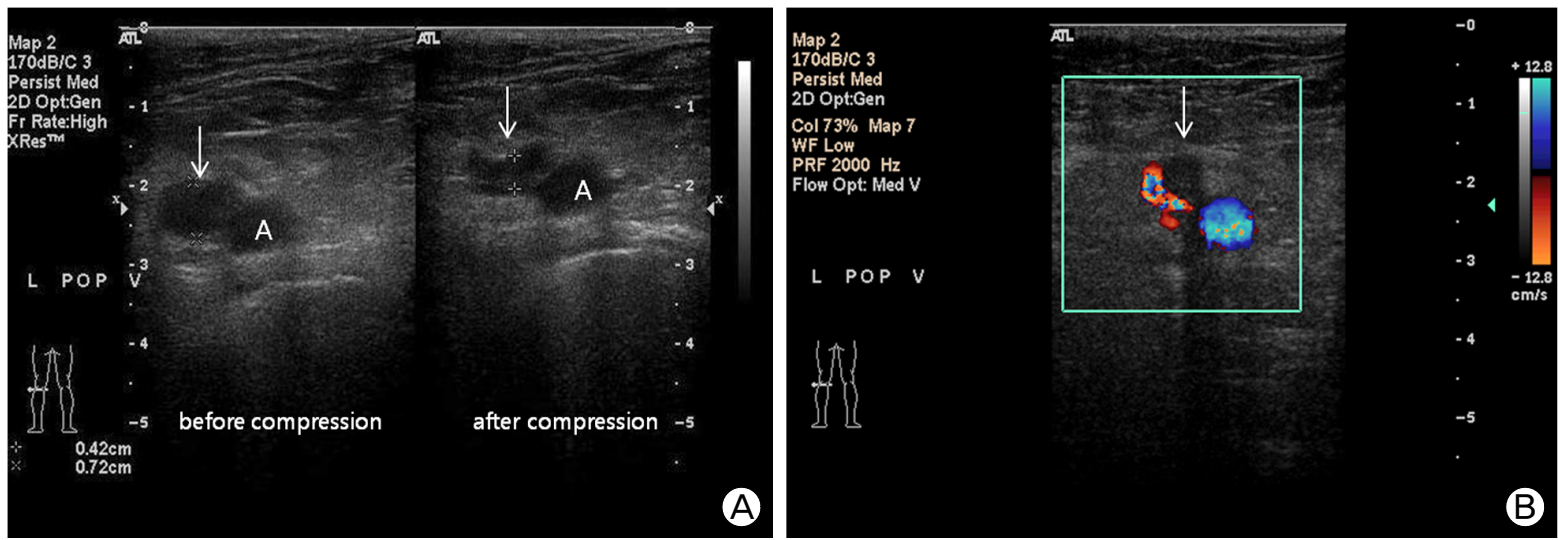

Fig. 2. Ultrasonography of lower extremities. Left popliteal vein (arrows) is not fully compressed after compression on transverse image at popliteal fossa (A). A hypoechoic lesion is seen as the flow void (arrow) within the left popliteal vein on transverse color Doppler image (B). These findings reveal a partial thrombosis at the left popliteal vein.

symptoms gradually worsened. She was transferred to the neurology department of our hospital and began antiplatelet therapy with 100-mg of aspirin once/day and 75-mg of clopidogrel hydrogen sulfate once/day. At the time of admission, her height was $155 \mathrm{~cm}$ and body weight was $66 \mathrm{~kg}$. Her medical history was remarkable for angina pectoris and hypertension. Her obstetric history included four pregnancies. On day 4 of hospitalization, she complained of severe coughing and developed a high fever of $38^{\circ} \mathrm{C}$ with dyspnea. She was treated immediately with 2-g of ceftriaxone once/day. She was assessed in the department of pulmonology on day 7 of hospitalization, and a computed tomography (CT) scan of the chest revealed a pulmonary embolism at the left interlobar artery and the right segmental artery accompanied by pulmonary infarctions and pneumonia at the right upper lobe, right middle lobe, and lingular seg- ment of the left upper lobe (Fig. 1). Doppler ultrasonography of the lower extremities revealed a partial thrombosis at the left popliteal vein with phlebedema of the left calf area (Fig. 2). She was started on LMWH for the pulmonary embolism and deepvein thrombosis $(3,800 \mathrm{IU} / 0.4 \mathrm{ml}$ nadroparin calcium twice/day, and subcutaneous injections in the arms on day 8 of hospitalization). Clopidogrel was discontinued at the same time.

On day 10 , she was transferred to the pulmonology department for further treatment of the pneumonia and pulmonary embolism. Her physical examination was unremarkable, except for a temperature of $37.7^{\circ} \mathrm{C}$, respiratory rate of $22 / \mathrm{min}$, coarse breath sounds, and bibasilar crackles with rhonchi on chest auscultation. Her oxygen saturation was $98 \%$ on $2 \mathrm{~L} / \mathrm{min}$ nasal $\mathrm{O}_{2}$. Peripheral blood testing performed upon her transfer to the department of pulmonology revealed anemia with a hemoglobin 

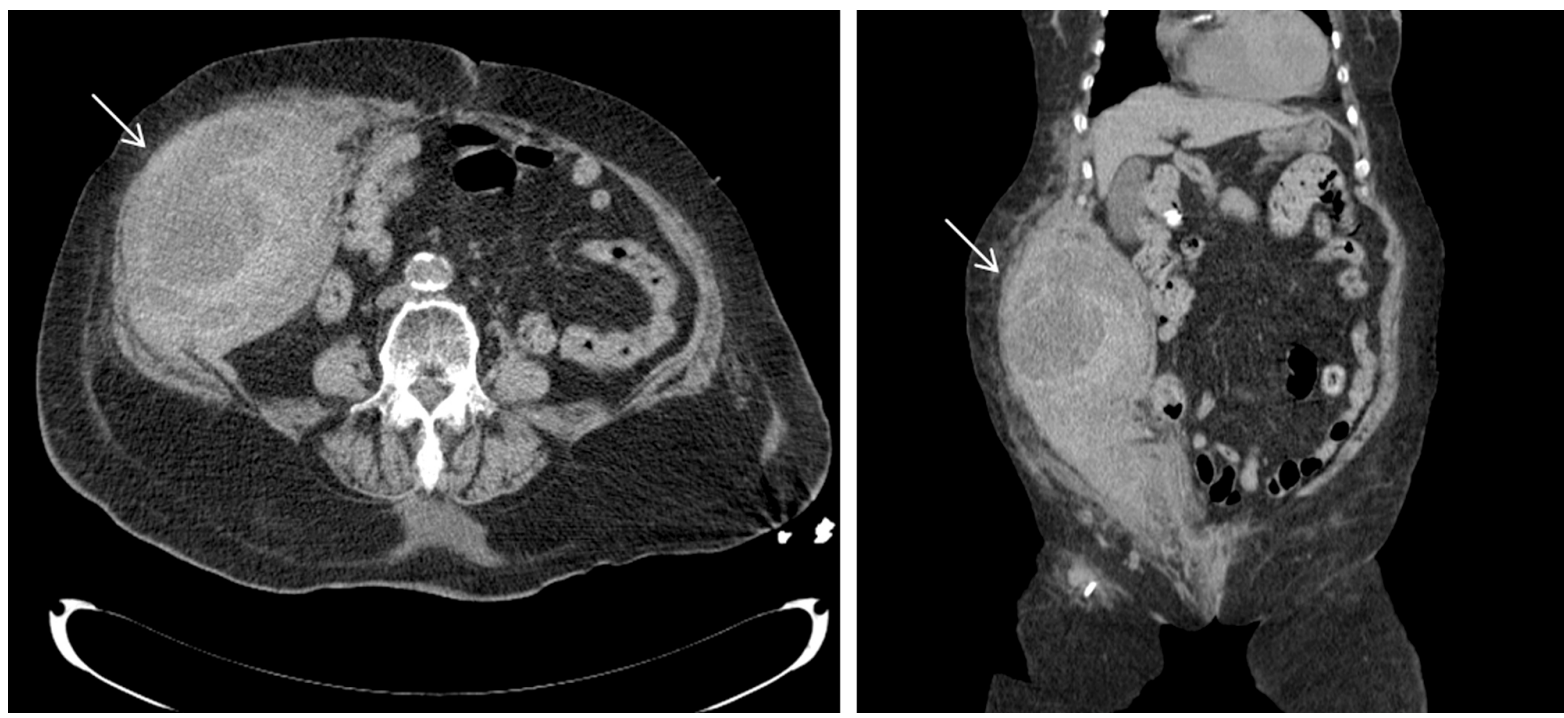

Fig. 3. Abdomen nonenhanced computed tomography (CT) scans. A CT scan of the abdomen demonstrated a large hematoma measuring $15 \times 10 \times 23 \mathrm{~cm}$ (arrows) in the right anterior abdominal wall muscles, including the transversus abdominis, internal oblique, external oblique, and rectus muscle without intraperitoneal bleeding. A large hematoma of peripheral high density and central low density was detected in the abdominal wall muscles.

level of $10.0 \mathrm{~g} / \mathrm{dl}$, hematocrit of $30.1 \%$, white blood cell count of $8,300 / \mathrm{mm}^{3}$ (39.0\% neutrophils), platelet count of $216,000 / \mathrm{mm}^{3}$, BUN level of $11 \mathrm{mg} / \mathrm{dl}$, creatinine level of $1.1 \mathrm{mg} / \mathrm{dl}$, and prothrombin time of $12.8 \mathrm{sec}$. Antibiotic therapy was changed to piperacillin sodium with tazobactam sodium combined with quinolone therapy, because she had developed intermittent fever. A serial follow-up chest X-ray showed slight worsening of the chest consolidation. Warfarin ( $5 \mathrm{mg}$ once/day) was added starting on day 8 of hospitalization.

However, after 5 days on LMWH (day 12 of hospitalization), the patient reported a sudden abdominal pain with distension. She subsequently became lethargic with a sudden drop in blood pressure to $70 / 40 \mathrm{mmHg}$, and her hemoglobin dropped significantly from $10.0 \mathrm{~g} / \mathrm{dl}$ to $6.2 \mathrm{~g} / \mathrm{dl}$. PT and INR levels were $32 \%$ and 2.09. A physical examination revealed a palpable mass and large ecchymosis in the right abdomen. A CT scan of the abdomen demonstrated a large hematoma measuring $15 \times 10 \times 23$ $\mathrm{cm}$ in the right anterior abdominal wall muscles, including the transversus abdominis, internal oblique, external oblique, and rectus muscle without intraperitoneal bleeding. A large hematoma of peripheral high density and central low density was detected in the abdominal wall muscles (Fig. 3). She was diagnosed with a large abdominal wall hematoma and treated with conservative medical care including immediate discontinuation of anticoagulants and administration of packed Red blood cells with fresh frozen plasma, along with fluid resuscitation and vitamin K. However, she developed shock, acute kidney injury with severe metabolic acidosis, and acute respiratory failure. The patient was placed on mechanical ventilation and was given vasopressor medication after being transferred to the intensive care unit. She was treated with continuous renal replacement therapy. Surgical intervention or endovascular embolization of bleeding vessels could not be performed because the patient was too unstable. She developed multiorgan failure rapidly, and eventually died the third day (day 14) after diagnosis of the large abdominal wall hematoma.

\section{DISCUSSION}

To our knowledge, this is the first reported fatal case of abdominal wall hematoma in an elderly female under LMWH use in Korea, although a case of severe bleeding in left thigh under LMWH use was reported in 2005.[8] LMWH can cause bleeding complications and sometimes leads to major life-threatening complications, as exemplified by the current case. An abdominal wall hematoma is an uncommon but serious bleeding complication associated with anticoagulant therapy. Over 30 cases of abdominal wall hematomas resulting from LMWH use have been reported.[5] However, an abdominal wall hematoma is clinically difficult to distinguish from other acute abdominal conditions and is usually diagnosed with an abdominal CT scan.[9] Clinical manifestations commonly include acute abdominal pain and abdominal wall ecchymosis, often combined with fever and vomiting, and a decrease in hemoglobin. These compli- 
cations may include features of hypovolemic shock, non-pulsatile abdominal mass, local peritoneal irritation signs, and abdominal compartment syndrome. $[5,10]$

LMWH is increasingly being used in both community and hospital settings to initiate anticoagulation for many clinical conditions, ischemic heart disease, deep vein thrombosis, and pulmonary emboli as well as for prophylaxis following hip and knee replacement surgery.[11] LMWH, which is a derivative of standard heparin, has an affinity for factor $\mathrm{X}$ and a high bioavailability, which allows patients to maintain therapeutic drug levels with subcutaneous injections once or twice/daily.[12] LMWH is at least as effective as unfractionated heparin and better than unfractionated heparin for reducing the risk of major bleeding and mortality.[13]

LMWH injections into the abdomen can directly damage the abdominal wall muscles, as well as the vasculature, and this is a common cause of hematomas associated with LMWH injections.[10] A hematoma can develop due to direct damage to the muscle or tearing in one of the superior or inferior epigastric vessels that run along its posterior aspect. Although our patient was injected subcutaneously with LMWH in the arm, a previous case showed that LMWH injections into the abdominal wall are not safe and are predominantly responsible for abdominal wall hematomas in the elderly.[1] Careful administration of this medication is warranted for patients with multiple bleeding risk factors. A review of other cases revealed that abdominal wall hematomas are found more commonly in females because they have less muscle mass and more-flaccid abdominal walls from pregnancies, with the highest incidence in females in their 50s.[10] The risk of bleeding increases in elderly patients and can cause serious complications, as in the present case. $[5,10]$ Other cases showed that a history of prior surgery, concomitant use of drugs such as antiplatelet or other anticoagulant agents in conjunction with LMWH, and renal failure could increase the risk of abdominal wall hematoma.[1,5,6] Straining the abdomen during processes such as coughing also increases the risk of hematoma formation, $[5,6,7]$ as the abdominal wall muscles suddenly contract to increase intrathoracic pressure. Repeated damage during cough attacks in this present case probably led to the abdominal wall hematoma, which was aggravated by the LMWH. The powerful contractions of the abdominal wall muscles may have caused the bleeding from anastomoses between the lower intercostal, lumbar, and ascending branch of the deep circumflex iliac artery. In our case, the patient had multiple risk factors, including being an elderly woman, history of multiple pregnancies with gravida 4 , and severe coughing due to pneumonia. In addition, piperacillin/tazobactam for the treatment of pneumonia may be considered as risk factor, in this case because it may induced platelet dysfunction, and an increased bleeding tendency when used with heparin.[14] The patient had taken nadroparin with antiplatelet agents and warfarin concomitantly.

In this case report, a large abdominal wall muscle hematoma, due to severe cough, occurred in a patient using LMWH for pulmonary embolism and deep vein thrombosis. Sudden abdominal pain together with an abdominal painful mass in severely coughing patients should alert physicians to a diagnosis of abdominal wall muscle hematoma, particularly in patients undergoing anticoagulant therapy, such as LMWH. Careful monitoring is required because this condition may be life threatening, particularly in elderly females using concomitant anticoagulation and in patients with abdominal straining.

\section{REFERENCES}

1) Kayrak M, Bacaksiz A, Yazici M: Is enoxaparin injection from the abdominal wall safe in elderly people?: a fatal case of rectus sheath hematoma. Can Fam Physician 2008; 54: 1246-8.

2) Agnelli G, Piovella F, Buoncristiani P, Severi P, Pini M, D'Angelo A, et al: Enoxaparin plus compression stockings compared with compression stockings alone in the prevention of venous thromboembolism after elective neurosurgery. $\mathrm{N}$ Engl J Med 1998; 339: 80-85.

3) Weitz JI: Low-molecular-weight heparins. N Engl J Med 1997; 337: 688-98.

4) Albertsen IE, Larsen TB, Rasmussen LH, Overvad TF, Lip GY: Prevention of venous thromboembolism with new oral anticoagulants versus standard pharmacological treatment in acute medically ill patients: a systematic review and metaanalysis. Drugs 2012; 72: 1755-64.

5) Nourbakhsh E, Anvari R, Nugent K: Abdominal wall hematoma associated with low-molecular-weight heparins: an important complication in older adults. J Am Geriatr Soc 2011; 59: 1543-5.

6) Barna L, Toth I, Kovacs E, Krizso E: Rectus sheath hematoma following exercise testing: a case report. J Med Case Rep 2009; 3: 9000.

7) Valdez G, Smalligan R: Clinical images: Spontaneous rectus sheath hematoma. CMAJ 2010; 182: E20.

8) Choi DH, Choi DW, Awe SI, Rhyu KH, Kim WJ, Kim YH, et al: Low-molecular-weight heparin associated intramuscular hematoma with compartment syndrome. Korean J Intern 
Med 2005; 69: S948-51.

9) Miyauchi T, Ischikawa M, Miki H: Rectus sheath hematoma in an elderly woman under anti-coagulant therapy. J Med Invest 2001; 48: 216-20.

10) Donaldson J, Knowles CH, Clark SK, Renfrew I, Lobo MD: Rectus sheath hematoma associated with low-molecular-weight heparin: a case series. Ann R Coll Surg Engl 2007; 89: 309-12.

11) Garcia DA, Baglin TP, Weitz JI, Samama MM; American College of Chest Physicians: Parenteral anticoagulants: antithrombotic therapy and prevention of thrombosis, 9th ed: American college of chest physicians evidence-based clinical practice guidelines. Chest 2012; 141(2 Suppl): e24S-43S.
12) Chuang YJ, Swanson R, Raja SM, Olson ST: Heparin enhances the specificity of antithrombin for thrombin and factor Xa independent of the reactive center loop sequence. Evidence for an exosite determinant of factor Xa specificity in heparin-activated antithrombin. J Bio Chem 2001; 276: 14961-71.

13) Krishnan JA, Segal JB, Streiff MB, Bolger DT, Eng J, Jenckes MW, et al: Treatment of venous thromboembolism with low-molecular-weight heparin: a synthesis of the evidence published in systematic literature reviews. Respir Med 2004; 98: 376-86.

14) Tatro DS: Drug interaction facts 2013: the authority on drug interactions. St. Louis, Lippincott Williams \& Wilkins. 2012, p 937. 